Apidologie, 1984, 15 (1), 11-22.

\title{
ÉTUDE PRÉLIMINAIRE DE LA DYNAMIQUE DES POPULATIONS D'UN PUCERON PRODUCTEUR DE MIELLAT : CINARA PECTINATAE NÖRDL. (HOMOPTERA-LACHNIDAE) DANS LE DOUBS. RELATION AVEC LA PLUVIOMÉTRIE
}

\author{
A. BLOC, Ph. THIEVENT et H. MONTAGNER \\ Laboraloire de Psychophysiologie, Faculté des Sciences et des Techniques \\ Route de Gray, Université de Franche-Comté, 25030 Besançon F.
}

\begin{abstract}
RESUME
Les résultats obtenus pendant 2 années consécutives montrent clairement qu'il y a une relation entre pluviométrie et dynamique des populations de Cinara pectinatae. Deux paramètres se dégagent : la quantité ou hauteur des pluies et le moment où celles-ci sont abondantes. On peut penser que, si ce dernier paramètre joue un rôle important, c'est parce qu'il affecte les populations de Cinara pectinatae à un moment clé du cycle biologique de l'espèce. Ainsi, lorsque les pluies sont abondantes durant la phase d'augmentation des premières générations de virgines, elles nuisent au bon développement des populations de Cinara pectinatae, condition indispensable à la production abondante des miellats, à partir desquels les miels de sapin sont produits dans la ruche.
\end{abstract}

\section{INTRODUCTION}

Recherchant les facteurs qui règlent le comportement de récolte des miellats de pucerons par l'abeille domestique Apis mellifica mellifica L., nous avons abordé l'étude de la dynamique des populations de pucerons de l'espèce Cinara pectinatae Nördl. sur le sapin Abies alba L. Les études ont été faites pendant deux années consécutives, 1981 et 1982 (elles ont été poursuivies en 1983 : en cours au moment de la rédaction définitive de cet article). Nous nous sommes particulièrement intéressés à l'influence éventuelle des conditions climatiques sur les fluctuations quantitatives des populations de Cinara pectinatae Nördl. On sait en effet que, parmi les facteurs qui interviennent dans la biocoenose des pucerons producteurs de miellats, la pluviométrie apparaît globalement comme l'un de ceux 
qui limitent la croissance des populations de Cinara pectinatae Nördl. Ainsi WüTherich (1977) montre, à partir d'une analyse des récoltes de miels de sapin et des données climatiques de 21 années consécutives, que les précipitations nuisent à la récolte des miellats, lorsqu'elles sont abondantes en automne, période de ponte chez ces pucerons, et en été pendant les miellées de sapin elles-mêmes. En revanche, LiEBig $(1978,1979,1980,1982)$, qui a suivi dans la nature des populations de Cinara pectinatae, n'observe pas de corrélation entre la dynamique des populations de puccrons et les facteurs climatiques. Selon lui, l'état physiologique de la plante-hôte paraît jouer un rôle essentiel.

Nous avons donc recherché nous-mêmes la part éventuelle des facteurs climatiques dans les fluctuations des populations de Cinara pectinatae.

\section{METHODE}

\section{Le comptage des pucerons}

La méthode de comptage des pucerons est la même que celle utilisée par LicBig en ForêtNoire (1977). Elle consiste à battre des branches de sapin de 10 à 50 ans d'âge au-dessus d'un carré de toile de $60 \mathrm{~cm} \times 60 \mathrm{~cm}$. Les pucerons tombent ainsi dans la toile, où ils sont dénombrés. Une série de 3 battages, portant sur 3 branches d'arbres différents, à moins de 3 mètres de hauteur, nous permet d'estimer la densité des pucerons par $\mathrm{m}^{2}$ de toile. Trois séries de 3 battages sont faits dans chaque station, qui représente une zone forestière couvrant une surface d'un hectare environ. Chaque branche est battue une seule fois au cours de la période de végétation. Cette méthode d'estimation autorise des comparaisons tout au long d'une même période de végétation, et d'une année à l'autre. En 1982, nous avons vérifié que le battage des branches sur une surface de $2 \mathrm{~m}^{2}$ donnait des résultats équivalents au battage de branches sur une surface de $3 \mathrm{~m}^{2}$ (très significatif à .01; coefficient de corrélation $\mathrm{r}$ : .934). Nous avons donc réduit l'aire minimum de battage à $2 \mathrm{~m}^{2}$. A ce stade de notre travail, il reste à évaluer l'efficacité de la méthode de battage pour les fondatrices de printemps (phase de développement de mars à mai) par rapport aux générations de virgines qui se succèdent jusqu'à l'automne, puis par rapport aux sexués.

\section{Lieux et périodes de comptage}

Situées dans les forêts des plateaux jurassiens, les stations de comptage ont été choisies en fonction de l'altitude et de la proximité d'une station permanente de la météorologie nationale. 5 stations se situent en forêt de la Joux (Levier : 700 à 800 mètres d'altitude) et 3 en forêt communale de Bonnevaux ( 900 à 1000 mètres d'altitude). Des observations plus irrégulières ont été faites dans d'autres stations (forêt du Mont d'Or, forêt du Risoux).

Les comptages sont faits toutes les 2 semaines, ou souvent toutes les semaines, du mois de mars au début du mois d'octobre, période pendant laquelle se développent les populations de Cinara pectinatae Nördl. (mars : éclosion des fondatrices; octobre : ponte des générations sexuées).

\section{Données climatologiques}

Elles sont recueillies par la station météorologique de Besançon qui publie tous les mois les relevés de ses stations réparties sur tout le département du Doubs (moyenne, minimum et maximum des températures et précipitations). Les données utilisées dans notre étude sont celles des stations de Levier, Bonnevaux, Mouthe et Chapelle-des-Bois, où se trouvaient nos ruches. 


\section{RÉSULTATS}

\section{La dymunique des populations de Cinara pectinatae}

Etablie par Likbig en 1979, et respectant les intervalles de temps donnés par celui-ci, la figure 1 représente schématiquement l'évolution temporelle des différents types d'individus qui, en Forêt-Noire (Allemagne Fédérale), composent les populations de pucerons appartenant à l'espèce Cinara pectinatae Nördl. Nos propres observations ne contredisent pas ce cycle annuel. Cependant, nous préciserons le cycle annuel de cette espèce à mesure que nous accumulerons les données obtenues par nos observations, faites à intervalles réguliers sur les mêmes sites.

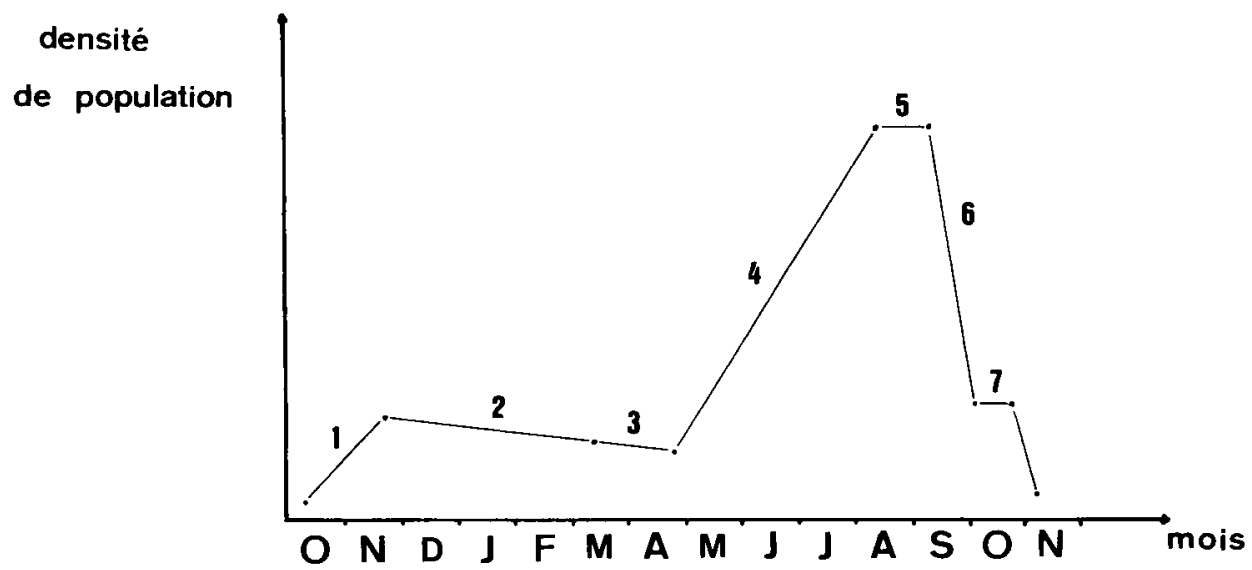

Fici. 1. - Représentation schématique de la dynamique des populations de Cinara pectinalae (Nördl.) au cours d'une année permettant un développement normal des populations (d'après Lisbig, 1979, modifié).

1. Ponte (octobre à novembre).

2. Hivernage des cufs (novembre à mars).

3. Phase de développement des fondatrices (mars à mai).

4. Phase de multiplication des premières générations (mai à juillet).

5. Phase de stagnation (juillet à août)

6. Chute des populations (août à septembre)

7. Générations sexuées.

Fici. 1. - Schematic representation of the population dynamics of Cinara pectinatae throughout the year (according to LIEBIG, 1979, modified).

1. Egg-laying (October to November).

2. Egg wintering (November to March).

3. Development period of fundatrices (March to May).

4. Growth period of the first asexual generations (May to July).

5. Stationary period (July, August).

6. Decrease of population (August, September).

7. Sexual forms (September, November). 
Les figures 2 à 5 montrent comment évoluent les populations de Cinara pectinatae dans 2 forêts du Haut-Doubs au cours des années 1981 et 1982. On observe que :

- le développement des populations est lent et tardif en 1981, à partir du $1^{\text {er }}$ juin, mais précoce et rapide en 1982, à partir du 15 mai. Les populations sont alors dans la phase 4 du cycle schématisé sur la figure 1 (phase de multiplication des premières générations de virgines) ;

- les populations diminuent parfois brusquement, à partir du $1^{\mathrm{cr}}$ juillet 1981 et au début de juin 1982. De telles diminutions n'apparaissent pas dans le schéma de la figure 1 ;

— la densité des individus tombe ou reste à un niveau très faible dans toutes les stations aux mêmes moments de l'année : le 22 juillet en 1981 et le $1^{\text {er }}$ juillet en 1982 ;

- la densité remonte nettement, souvent de façon linéaire, en août 1981. Cette augmentation est suivie dans tous les cas d'une miellée de sapin à partir du 20 août et d'une récolte des miellats par l'abeille domestique jusqu'au 10 septembre ;

- en revanche, en 1982, la reprise de croissance des populations, observée dans la première moitié de juillet, est suivie d'un effondrement. Les populations tendent alors à disparaître ou restent à un niveau très faible à la fín du mois de juillet et en août.

En 1981 et en 1982, les courbes d'évolutions des populations de Cinara pectinatae Nördl. sont donc différentes de la courbe établie par LiEBig en 1979. Elles sont notamment marquées par une chute brusque et significative du niveau des populations à 2 moments différents du cycle biologique de cette espèce.

Par ailleurs, au cours de la $2^{\prime \prime}$ quinzaine de juillet 1981 et de la $1^{\text {re }}$ quinzaine de juin 1982, la densité de pucerons ne dépasse pas le niveau de 40 pucerons par $\mathrm{m}^{2}$, alors que les densités observées en Forêt-Noire par Lıicbig de 1977 à 1980 atteignent des valeurs de 80 à 140 pucerons par $\mathrm{m}^{2}$ (ces années ont aussi été marquées par une forte miellée de sapin).

\section{Les facteurs climatiques et la dynamique des populations de puccrons}

Les figures 2 à 5 montrent comment ont évolué la pluviométrie et la dynamique des populations de Cinara pectinatae au cours du printemps et de l'été, en 1981 et en 1982. Les écoclimatogrammes (MesquidA, 1975) des figures 6 et 7 représentent globalement le climat des années 1981 et 1982, en prenant en compte à la fois la pluviométrie et la température. Ils permettent une représentation synthétique de la physionomie apicole des stations étudiées et peuvent être comparés aux figures 2 et 3 qui retracent dans les mêmes stations le développe- 


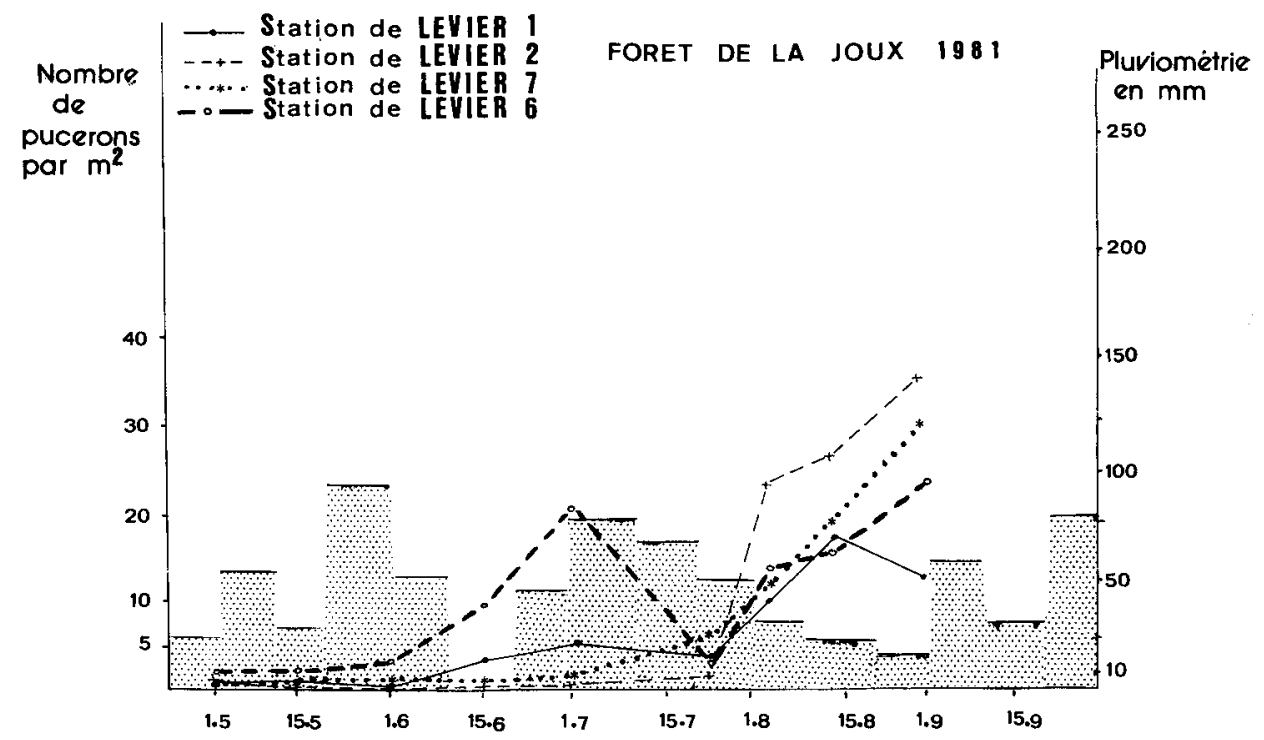

FIG. 2. - Dynamique des populations de Cinara pectinatae dans la forêt de la Joux durant l'été 1981, superposée à la pluviométrie (celle-ci est figurée par des colonnes de points).

FIG. 2. - Population dynamics of Cinara pectinatae in the Joux forest throughout late Spring and Summer 1981, in relation to rainfall (dotted area).

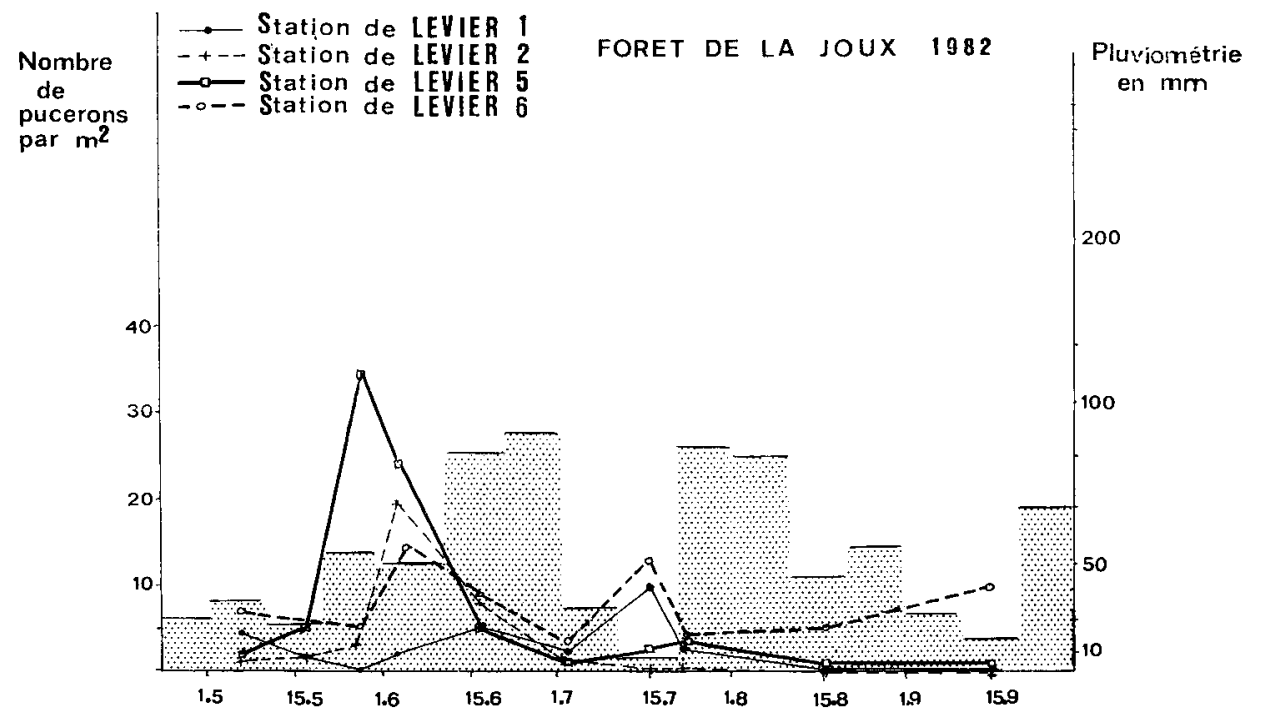

Fıg. 3. - Dynamique des populations de Cinara pectinatae dans la forêt de la Joux durant l'été 1982, superposée à la pluviométrie (celle-ci est figurée par des colonnes de points).

FIG. 3. - Population dynamics of Cinara pectinatae in the Joux forest throughout late Spring and Summer 1982, in relation to rainfall (dotted area). 
FORET DE BONNEVAUX 1981

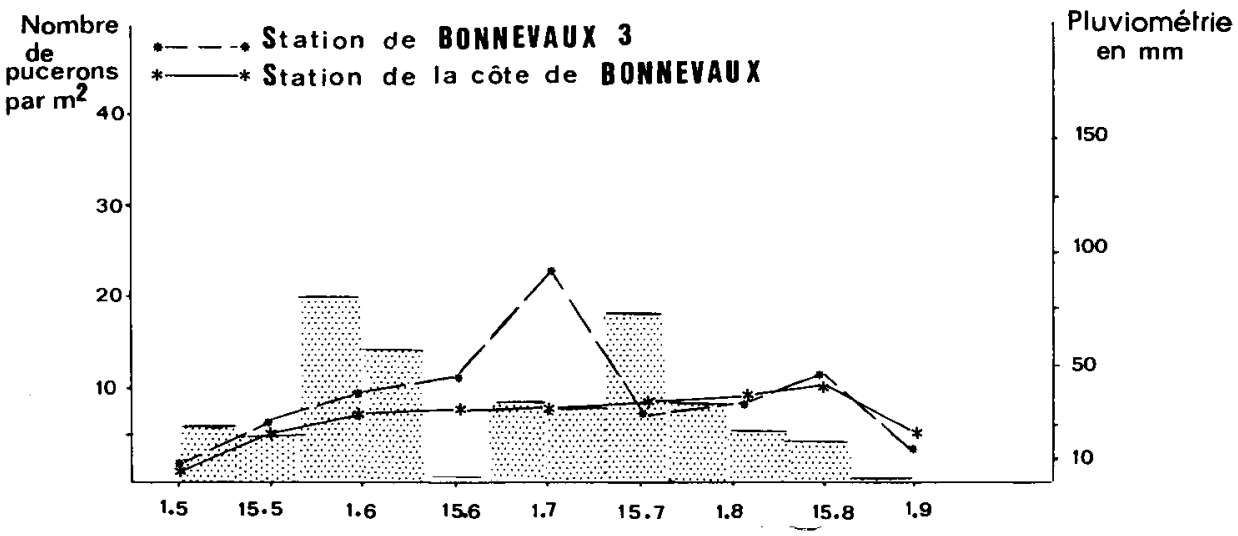

FIG. 4. - Dynamique des populations dans la forêt de Bonnevalux daramt léré 198I. superposée à la pluriométrie (celle-ci est figurée par des colonnes de points).

FIG. 4. - Population dyanics of Cinara pectinatae in the Bonnevaux forest throughout late Spring and Summer 1981 , in relation to rainfall (dotted area).

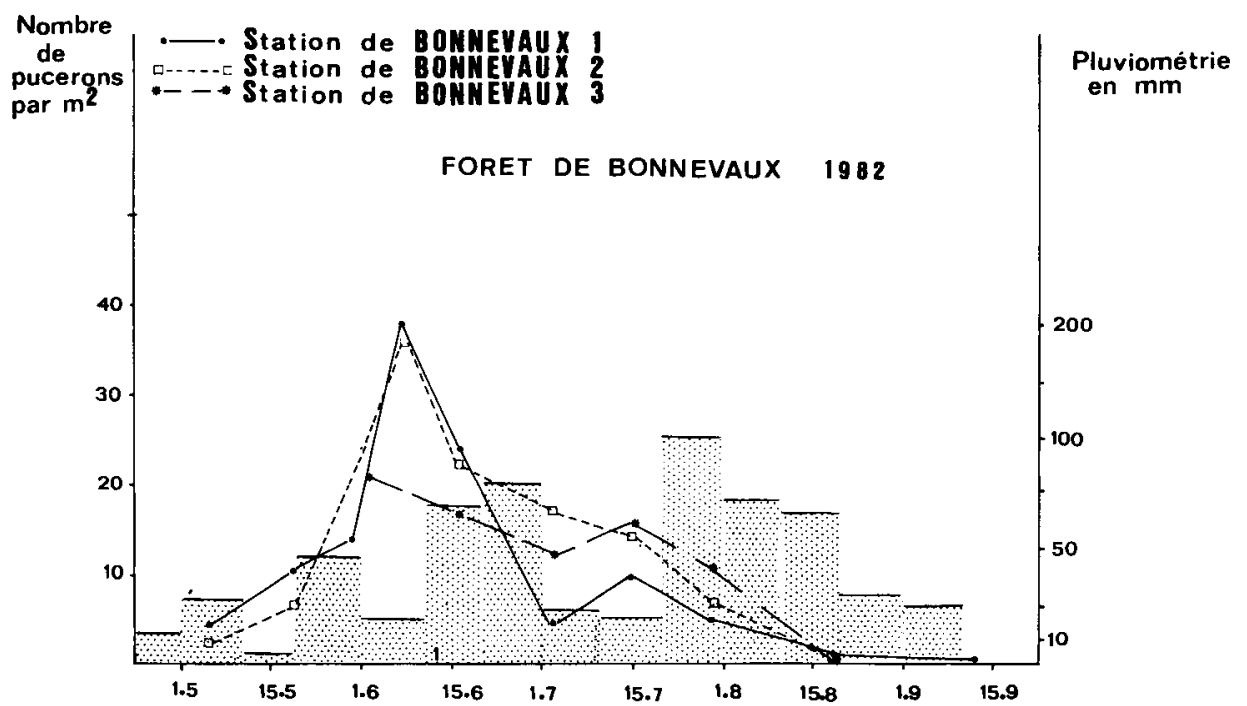

Fici. 5. - Dynamique des populations dans la forêt de Bonnevaux durant l'été 1982, superposée à la plaviométrie (celle-ci est figurée par des colonnes de points).

FIG. 5. - Population dymamics of Cinara pectinatae in the Bonnevaux forest throughoul late Spring and Summer 1982, in relation to rainfall (dotted area). 
ment des populations de Cinara pectinatae. Ces écoclimatogrammes font notamment ressortir le caractère pluvieux et humide de juillet 1981 et de juin, juillet et août 1982 .

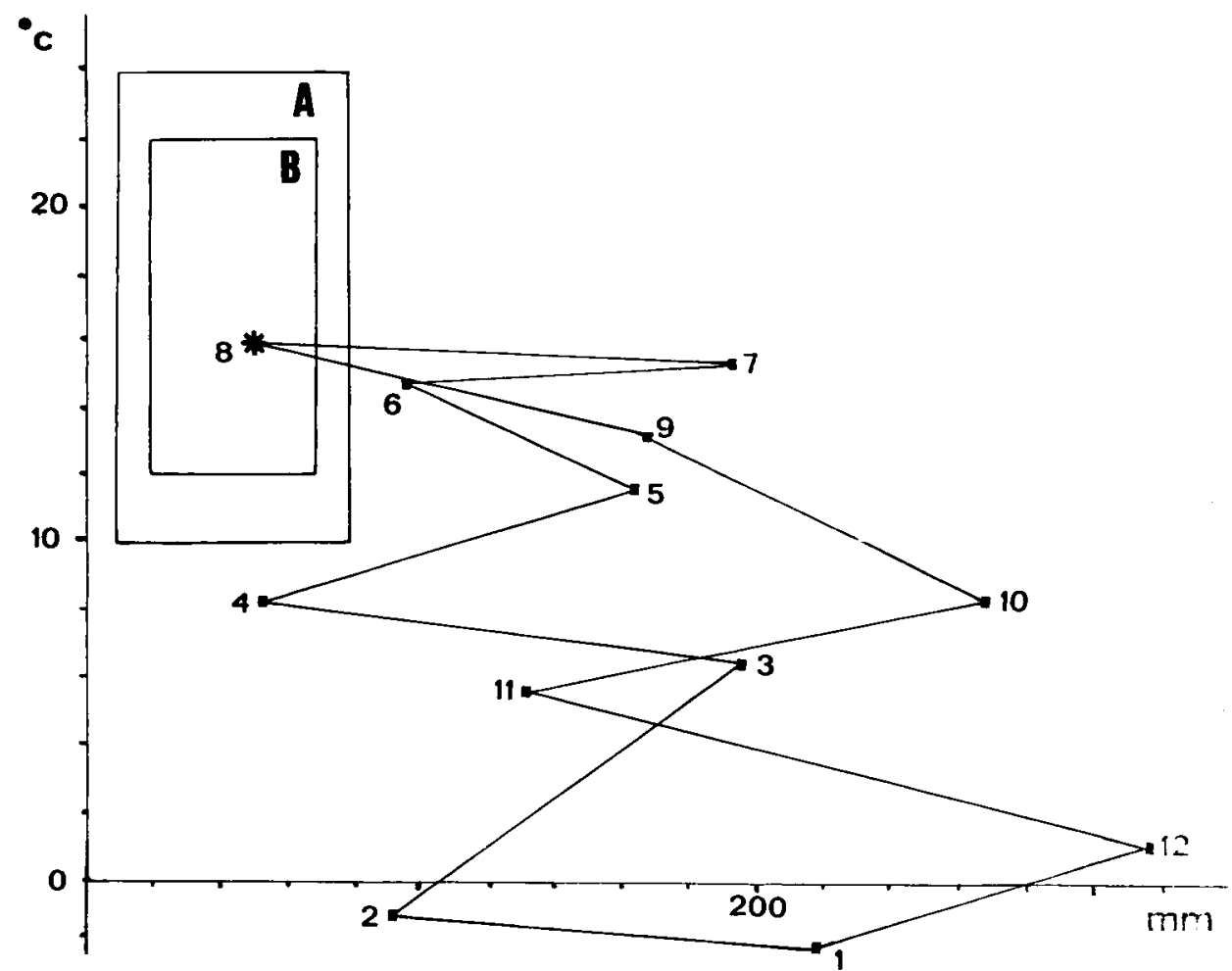

FIG. 6. - Ecoclimatogramme des slations de Levier en 1981.

A : Conditions climatiques extrêmes qui sont favorables à l'activité normale des butineuses.

B : Conditions météorologiques optimales qui sont favorables aux miellées et aux récoltes par les abeilles.

* : Miellée de sapin.

Les chiffres de 1 à 12 représentent les mois (1: janvier ; 12 : décembre).

En ordonnées : température moyenne mensuelle en " $C$.

En abscisses : précipitations totales mensuelles en $\mathrm{mm}$.

FIG. 6. - Euoclimalogram in Levier region in 1981.

A : Levels of Temperature and rainfall which allow a normal activity of bees.

B : Levels of temperature and rainfall which are the best for honey flow and foraging activity of bees.

* : Honeydew in fir trees.

The numbers 1 to 12 symbolize months (1: January; 12 : December).

$\mathrm{Y}$-axis : monthly mean temperature in " $\mathrm{C}$.

$\mathrm{X}$-axis : monthly rainfall in $\mathrm{mm}$. 


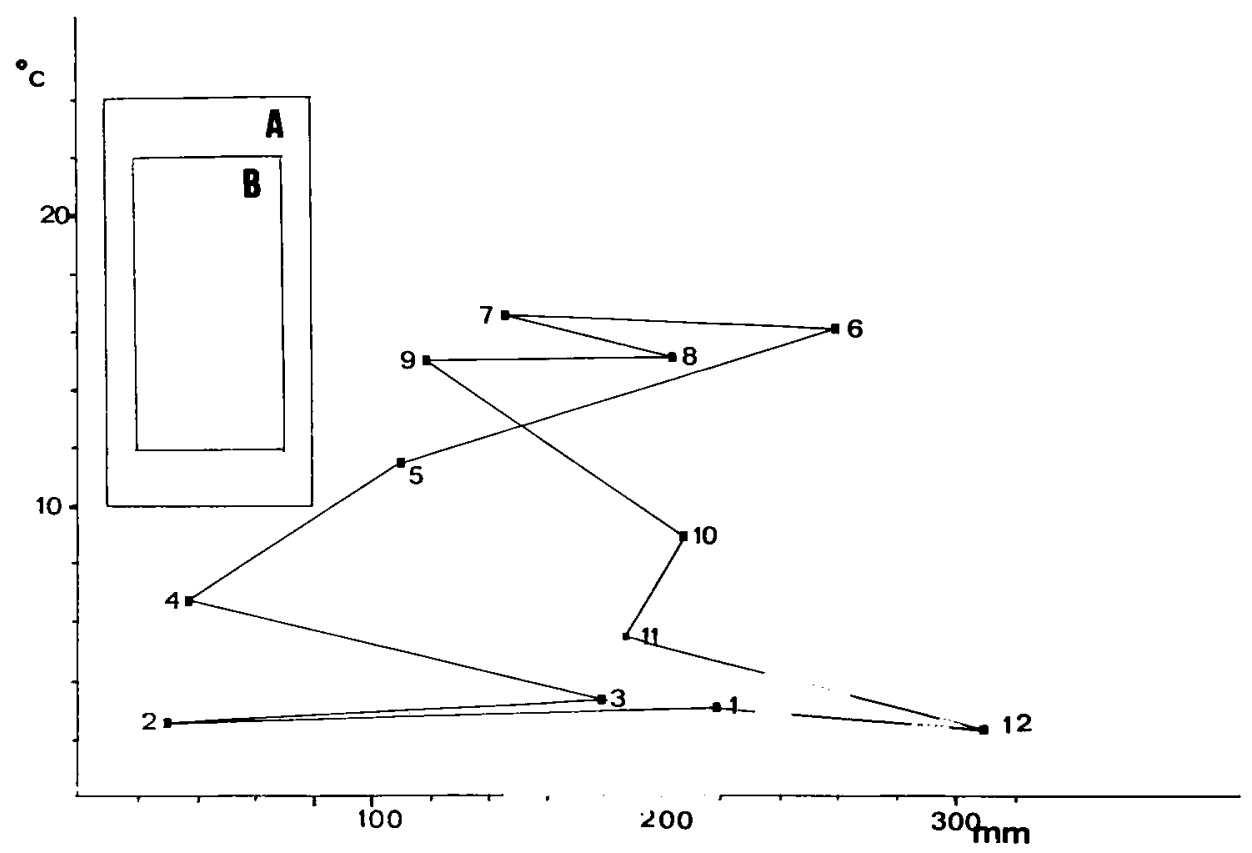

Fig. 7. - Ecoclimatogramme des stations de Levier en 1982. Même légende que Fig. 6.

FIG. 7. - Ecoclimatogram in Levier region in 1982. As in Fig. 6.

Cependant, si les années 1981 et 1982 ont été marquées par des périodes de forte pluviosité, c'est à des moments différents : juillet en 1981, juin et août en 1982. Parallèlement, la production des miellats et des miels de sapin a été très différente au cours de ces 2 années : il y a eu des miellées de sapin, parfois abondantes, à la fin du mois d'août 1981 ; il n'y en pas eu en août 1982. En ce qui concerne l'année 1982, la pluviométrie élevée au cours de la 3" décade de mai et au début de juin, a touché les fondatrices et les premières générations de virgines susceptibles d'engendrer une descendance importante. Or, on sait que les femelles les plus fécondes appartiennent précisément à ces premières générations (LEONARIT, 1940 ; Maquelin, 1974). De plus, le nombre de générations de virgines n'est que de 4 à 5 entre 600 et 1000 mètres d'altitude (MAQuelin, 1974), contre 8 à 10 à 300 mètres (Geinitz, 1930 ; LeonardT, 1940), ce qui affecte évidemment le potentiel reproducteur des populations vivant aux altitudes choisies dans notre étude (700 à 1000 mètres). Par conséquent, une chute de population au début du cycle annuel de Cinara pectinatae paraît affecter de façon marquée la dynamique des populations au cours de l'été. 
Par comparaison, des conditions climatiques peu favorables au printemps (1981) empêchent un accroissement rapide des populations, en retardant l'éclosion des œufs, ce qui rend peut-être les fondatrices (apparition et développement de mars à mai) et les premières virgines (multiplication des premières générations en mai-juin) moins vulnérables à une brusque dégradation de la climatologie, moins sévère au début de l'été qu'au printemps (WüTHERICH, 1977). L'observation en juillet 1982 ( $2^{\circ}$ quinzaine) d'une forte miellée dans les forêts de sapins au pied du Mont-d'Or (1100-1200 mètres, département du Doubs), combinée à la présence d'une population de Cinara pectinatae dont la densité était d'environ 40 individus par $\mathrm{m}^{2}$ en moyenne, conforte cette hypothèse. En effet, dans cette région, les conditions climatiques de l'hiver se sont prolongées en avril et mai, ce qui a retardé l'apparition des premières fondatrices, puis des premières virgines, et permis ensuite aux générations à potentiel reproducteur important de se multiplier, dès que les conditions climatiques sont devenues favorables.

\section{DISCUSSION ET CONCLUSIONS}

La comparaison deux à deux des courbes des figures 2 et 3 par le test du X2 montre que l'évolution des populations de Cinara pectinatae ne se fait pas au hasard dans les stations étudiées. L'analyse détaillée fait en effet apparaître des corrélations entre dynamique des populations de Cinara pectinatae et pluviométrie, en 1981 et en 1982. Ainsi, l'étude de la pluviométrie montre-t-elle que, en avril et mai 1982, celle-ci est inférieure de $30 \%$ à celle de la même période en 1981, de $65 \%$ (avril 1982) et $20 \%$ (mai 1982) à celle de la moyenne des 10 dernières années, Parallèlement, on observe en 1982 une croissance précoce de la plupart des populations de pucerons. Le faible niveau des pluies de la période avril-mai 1982 a permis le bon développement des phases 3 (développement des fondatrices) et 4 (multiplication des premières générations de virgines) de la figure 1 .

Le mois de juin 1982 est marqué par une forte pluviométrie $(260,6 \mathrm{~mm}$ contre $96,9 \mathrm{~mm}$ en 1981 et $146 \mathrm{~mm}$ pour la moyenne des 10 dernières années) et de violents orages de grêle. En même temps, le niveau des populations de Cinara pectinatae s'effondre à une moyenne de 2,5 individus par $\mathrm{m}^{2}$, après avoir atteint un maximum le 27 mai et le 3 juin. De même, à la mi-juillet 1982, les populations tombent à une moyenne de 7 individus par $\mathrm{m}^{2}$, alors que la pluviométrie atteint un niveau élevé $(191,8 \mathrm{~mm}$ contre $114,9 \mathrm{~mm}$ pour la moyenne des 10 dernières années).

L'analyse de la pluviométrie par décade en juillet $1982(31 \mathrm{~mm}$ pour la 
première décade, $8 \mathrm{~mm}$ pour la deuxième, $105 \mathrm{~mm}$ pour la troisième) permet d'expliquer la nouvelle chute des populations à partir de la deuxième moitié de juillet. Il n'y aura plus ensuite de croissance des populations.

Deux hypothèses au moins peuvent être formulées pour rendre compte de l'influence de la pluviométrie sur la limitation des populations de Cinara pectinatae :

1. les fortes pluies, la grêle et les orages entraînent une forte mortalité en agissant directement sur les pucerons. C'est ce que tendent à montrer les comptages de pucerons réalisés de 24 à 48 heures après un orage : au cours des battages, la proportion des pucerons morts qui tombent sur la toile peut s'élever à $60 \%$;

2. Ies précipitations agissent sur le sol et la plante-hôte, en modifiant notamment la qualité de la sève élaborée, à partir de laquelle se nourrissent les pucerons, et la pression osmotique au sein des rameaux de sapin (Chararas, 1979).

Cependant, la dynamique des populations de Cinara pectinatae n'est pas toujours corrélée avec la pluviométrie. Par exemple, si on compare les 2 stations de Bonnevaux que nous avons étudiées en 1981, la densité moyenne des pucerons reste la même du 15 juin au $1^{\text {cr }}$ juillet dans la station de la Côte de Bonnevaux alors que, dans le même temps, elle double dans la station de Bonnevaux 3. Des différences importantes existent aussi entre les 4 stations de Levier étudiées en 1981 : inférieure à 5 individus par $\mathrm{m}^{2}$ le $1^{\text {er }}$ juin dans toutes les stations, elle passe à plus de 20 le $1^{\text {cr }}$ juillet à Levier 6 et reste à un faible niveau dans les 3 autres stations. Il en va de même en 1982. Par exemple, à la fin du mois de mai, la densité des pucerons est supérieure à 30 individus par $\mathrm{m}^{2}$ à Levier 5 , alors qu'elle est respectivement de 5 , moins de 5 et 0 à Levier 6 , Levier 2 et Levier 1 .

De telles différences peuvent expliquer que Liebig et SchlipF (1982) n'aient pas trouvé de corrélation entre la densité des populations de Cinara pectinatae et la pluviométrie.

Plusieurs hypothèses peuvent être avancées pour rendre compte des différences que nous avons observées entre les stations d'un même site :

1. les prédateurs de pucerons étant nombreux (larves de Chrysops; Coccinellidae ; Ichneumonidae ; Syrphidae ; etc.), on peut penser que ce facteur intervient très localement, au niveau d'un groupe d'arbres, alors qu'il est moindre pour un autre groupe d'arbres du même site ;

2. parfois très localisés en montagne, les orages peuvent affecter les arbres à des degrés divers, selon la pente, le versant, l'exposition aux pluies ou le degré de protection fourni par l'ensemble de la couverture végétale ;

3. les pluies peuvent affecter différemment la physiologie des arbres qui 
abritent les populations de pucerons, selon l'âge des arbres et la nature des associations végétales ;

4. Ies pluies peuvent affecter différemment les populations de pucerons elles-mêmes, selon la composition de celles-ci (rapport larves/imagos; rapport fondatrices/virgines; etc.). Les études des groupements et sociétés d'insectes montrent en effet clairement que le développement imaginal, le comportement, la physiologie et la mortalité des individus présentent parfois des différences importantes selon la composition du groupement ou de la société, et par rapport aux conditions autécologiques.

Il nous appartiendra de cerner la part respective de ces facteurs au sein d'un même site et d'un même biotope, dans la limitation de la croissance des populations de pucerons, ceci tout au long du cycle annuel de Cinara pectinatae. Nous avons vu en effet que les précipitations avaient des effets différents selon le moment du cycle annuel où elles s'exercent.

Reçu pour mublication en mars 1983. Accepté pour publication en jamior 1984.

\section{SUMMARY}

PREIIMINARY DATA ON POPULATION DYNAMICS OF A HONEYDEW-PRODUCING APHID, CINARA PECTINATAE NÖRDL. (HOM. LACHNIDAE) IN THE DOUBS AREA (FRANCE) - RELATIONSHIP TO RAINFALI,

The population dynamics of the aphid Cinara pectinatae Nördl. were determined by successive samplings from March to October in fir forests of the Jura area (eastern France). The method consisted of beating fir branches and allowing the aphids to fall on a $60 \times 60 \mathrm{~cm}$ cloth held below.

After having studied Cinara pectinatae populations for two years, we found a significant correlation between their dynamics and rainfall (Fig. 2-5). Two parameters appeared to be important : the rainfall level and the time when it was heavy. If the latter parameter plays an important role, it is presumably because it affects populations of Cinara pectinatae at a key moment of their biological cycle. Thus, when rainfall was heavy during the development of the first virginipar generations, it prevented Cinara pectinatae populations from reaching high levels. This in turn prevented heavy honeydew production and a «fir honey» harvest by bees.

\section{ZUSAMMENFASSUNG}

EINLEITENDE STUDIEN ZUR POPULATIONSDYNAMIK

VON CINARA PECTINATAE NÖRDL. (HOMOPTERA, LACHNIDAE) IM DOUBS. ABHANGIGKEIT VON DER NIEDERSCHLAGSMENGE

Die Populationsdynamik von Cinara pectinatea (Nördl.) im Tannenwald des Jura-Plateaus wurde. durch sukzessive Probennahme von März bis Oktober untersucht. Die Proben der Läuse erhielt man. durch Schlagen der Tannenzweige über einem Tuch (Größe $60 \times 60 \mathrm{~cm}$ ). 
Die in zwei aufeinanderfolgenden Jahren erhaltenen Ergebnisse zeigen deutlich, daß eine Beziehung zwischen den Niederschlägen und der Populationsdynamik von Cinara pectinatae besteht (Fig. 2-5). Dabei traten zwei Parameter der Pluviometrie besonders hervor : Die Niederschlagsmenge oder -höhe und der Zeitpunkt gehöufter Niederschläge. Die wichtige Rolle des letztgenannten Parameters ist möglicherweise darauf zurückzuführen, daß er in einem entscheidenden Moment in den Biozyklus von Cinara pectinatae eingreift. Reichliche Niederschläge während der Vermehrungsphase der ersten parthenogenetischen Generationen schaden einer positiven Populationsentwicklung von Cinara pectinatae, die jedoch eine unverzichtbare Voraussetzung für eine reichliche Honigtauproduktion ist, auf der die Produktion des Tannenhonigs im Bienenstock basiert.

\section{BIBLIOGRAPHIE}

Chararas C., 1979. - Ecophysiologie des insectes parasites des forêts. Edité par l'auteur, 38, av. R.-Coty, 75014 Paris.

Geinitz B., 1930. — Die Entstehung des Tannenhonigs. Arch. Bienenkd., 9, 308-318.

LEONHARDT H., 1940. - Beiträge zur Kenntnis der Lachniden der wichtigsten Tannenhonigtauerzeuger. Z. ang. Entomol., 27, 208-281.

Liebig G., 1978. - Eine Analyse der Tannentracht 1977 und 1978. Die Bienenpflege, 12, $243-250$.

Liebig G., 1979. - Hinweise für die Beobachtung der Tannentracht. Allg. dtsch. Imkerztg., 1, 65-67 ; $2,101-103 ; 3,129-131 ; 4,204-206$.

Liebig G., 1980. - Wie sind die Aussichten für eine Tannentracht 1980. Die Bienenpflege 4, 74-78.

Liebig G., Schtipf U., 1982. - Was war los mit der Tannentracht 1982. Allg. disch. Imkerztg., 372-375.

Liebig G., Schlpf U., Düwel W., 1982, - - Witterungsverlauf und Massenwechsel der grünen Tannenhoniglaus Cinara pectinatae (Nördl.) (Homoptera, Lachnidae) in der Jahren (1977-1981). Apidologie, 13, 275-295.

Maquelin C., 1974. - Observation sur la biologie et l'écologie d'un puceron utile à l'apiculture, Buchneria pectinatae (Nördl.) (Homoptera, Lachnidae). Thèse E.T.H., Zürich, 118 p.

Mesquida J., 1975. - Application des écoclimatogrammes pour la représentation synthétique de la physionomie apicole d'une région. Apimondia, $25^{\circ}$ Congrès International d'Apiculture, 499-507.

Wütherich G., 1977. - Untersuchungen über Honigtau, insbesondere über die Witterungsabhängigkeit des Massenwechsels Honigtau erzeugender Lachniden und die daraus resultierenden guten und schlechten Honigjahre. These Forstzoologisches Institut der Universität Freiburg, 114 p. 